\title{
Whole-methylome analysis of circulating monocytes in acute diabetic Charcot foot reveals differentially methylated genes involved in the formation of osteoclasts
}

\author{
Jennifer Pasquier ${ }^{\ddagger 1,2,3}$, Mark Spurgeon $¥, 3,4$, Martina Bradic ${ }^{3}$, Binitha Thomas ${ }^{1}$, Amal \\ Robay ${ }^{1,3}$, Omar Chidiac1, Marie-Joe Dib¹, Rebal Turjoman¹, Alexandra Liberska5, Michelle \\ Staudt $^{3}$, Khalid A Fakhro ${ }^{1,6}$, Robert Menzies ${ }^{7}$, Amin Jayyousi ${ }^{8}$, Mahmoud Zirie ${ }^{8}$, Jassim Al \\ Suwaidi ${ }^{9}$, Rayaz A Malik ${ }^{10}$, Talal Talal7, Arash Rafii ${ }^{2,3}$, Jason Mezey ${ }^{3,4}$, Juan \\ Rodriguez-Flores ${ }^{3,4}$, Ronald G Crystal ${ }^{3}$ \& Charbel Abi Khalil*,1,3,9,10 \\ ${ }^{1}$ Epigenetics Cardiovascular Laboratory, Department of Genetic Medicine, Weill Cornell Medicine-Qatar, Doha, Qatar \\ ${ }^{2}$ Stem Cell and Microenvironment Laboratory, Weill Cornell Medicine-Qatar, Doha, Qatar \\ ${ }^{3}$ Department of Genetic Medicine, Weill Cornell Medicine, NY, NY-10021, USA \\ ${ }^{4}$ Department of Biological Statistics and Computational Biology, Cornell University, Ithica, NY, NY-14850, USA \\ ${ }^{5}$ Flow Cytometry Facility, Microscopy Core, Weill Cornell Medicine-Qatar, Doha, Qatar \\ ${ }^{6}$ Department of Human Genetics, Sidra Medical and Research Center, Doha, Qatar \\ ${ }^{7}$ Department of Podiatry, Hamad Medical Corporation, Doha, Qatar \\ ${ }^{8}$ Department of Diabetes and Endocrinology, Hamad Medical Corporation, Doha, Qatar \\ ${ }^{9}$ Heart Hospital, Hamad Medical Corporation, Doha, Qatar \\ ${ }^{10}$ Joan and Sanford I. Weill Department of Medicine, Weill Cornell Medicine, NY, NY-10021, USA \\ *Author for correspondence: cha2022@med.cornell.edu \\ ${ }^{\ddagger}$ Authors contributed equally
}

Aim: To assess whether DNA methylation of monocytes play a role in the development of acute diabetic Charcot foot (CF). Patients \& methods: We studied the whole methylome (WM) of circulating monocytes in 18 patients with Type 2 diabetes (T2D) and acute CF, 18 T2D patients with equivalent neuropathy and 18 T2D patients without neuropathy, using the enhanced reduced representation bisulfite sequencing technique. Results \& conclusion: WM analysis demonstrated that CF monocytes are differentially methylated compared with non-CF monocytes, in both CpG-site and gene-mapped analysis approaches. Among the methylated genes, several are involved in the migration process during monocyte differentiation into osteoclasts or are indirectly involved through the regulation of inflammatory pathways. Finally, we demonstrated an association between methylation and gene expression in cis- and trans-association.

First draft submitted: 5 September 2018; Accepted for publication: 16 October 2018; Published online: 1 November 2018

Keywords: Charcot foot $\bullet$ diabetes $\bullet$ DNA methylation $\bullet$ epigenetics $\bullet$ epigenomics $\bullet$ gene expression $\bullet$ genetics

Charcot foot (CF) disease is a devastating complication of diabetes, associated with an increased risk of soft tissue infections, foot ulcers and amputations [1]. It is characterized by an exaggerated bone resorption [2], believed to be induced by an increased numbers of osteoclasts and their activity [3,4]. Osteoclasts are derived from monocytes, mostly from $\mathrm{CD}_{14}{ }^{+}$that have the highest potential to differentiate, following a differentiation pathway that results in mature functional osteoclasts whose role is to activate bone resorption [5].

Epigenetics modulates the differentiation of many adult cell types from the progenitor or primary cells with whom they share the same DNA sequence, and plays an important role in gene transcription through three major components: DNA methylation, noncoding RNAs and post-translational changes of histone proteins [6]. Methylome, which is the set of nucleic acid methylation modifications in an organism's genome or in a particular cell [7], also participate in the pathophysiology of several diseases by controlling cellular differentiation processes and transcriptional activities of genes [8]. Therefore, we hypothesized that the methylome of circulating monocytes in patients with acute diabetic CF could be involved in the pathogenesis of the disease. 
Table 1. Baseline characteristics of the participants included in the study.

\begin{tabular}{|c|c|c|c|c|}
\hline Baseline characteristics & Diabetes with Charcot $(n=18)$ & $\begin{array}{l}\text { Diabetes with neuropathy ( } \mathrm{n} \\
=18 \text { ) }\end{array}$ & $\begin{array}{l}\text { Diabetes without neuropathy } \\
(n=18)\end{array}$ & p-value \\
\hline Gender (M/F) & $13 / 5$ & $13 / 5$ & $13 / 5$ & - \\
\hline HbA1c (\%) & $8.1(1.7)$ & $8.3(2.2)$ & $8.1(1.7)$ & 0.88 \\
\hline Fasting glucose (mmol/l) & $9.1(4.6)$ & $10(4.5)$ & $9.9(3.9)$ & 0.79 \\
\hline Diastolic BP (mmHg) & $74(7.6)$ & $73(9.7)$ & $78(9.3)$ & 0.21 \\
\hline eGFR (ml/min) & $77(32.7)$ & $81(37.6)$ & $101(22.6)$ & 0.07 \\
\hline WBC $\left(\times 10^{3} / \mu \mathrm{I}\right)$ & $8.8(4.2)$ & $7.3(1.6)$ & $8.04(2.2)$ & 0.36 \\
\hline Monocytes (\%) & $7(1.7)$ & $6(2.4)$ & $6.9(1.4)$ & 0.21 \\
\hline
\end{tabular}

Data are represented as mean (standard deviation). $\mathrm{p}$-values were calculated with ANOVA test. Each group has $\mathrm{n}=18$ and patients are matched for age, gender, BMl and $\mathrm{HbA} 1_{\mathrm{c}}$ across all groups such that these covariates were not significantly different among groups (rightmost column).

BMI: Body mass index; BP: Blood pressure; eGFR: Estimated glomerular filtration rate; F: Female; M: Male; WBC: White blood cell count.

In this study, we report the presence of differentially methylated genes involved in migration, differentiation and formation of osteoclasts in circulating monocytes of Type 2 diabetes (T2D) patients with acute CF, compared with T2D patients without CF.

\section{Patients \& methods}

Subjects

$18 \mathrm{~T} 2 \mathrm{D}$ patients with acute $\mathrm{CF}$, matched for age, gender, $\mathrm{BMI}$ and $\mathrm{HBA}_{1 \mathrm{C}}$, with $18 \mathrm{~T} 2 \mathrm{D}$ patients with neuropathy but no CF and 18 T2D without neuropathy or CF were studied (Table 1). CF patients were recruited from the podiatry clinic at Hamad Medical Corporation (HMC), Doha, Qatar. All other patients were recruited from the department of endocrinology and diabetes at HMC.

Acute CF was diagnosed according to the American Diabetes Association and the American Podiatric Medical Association task force [1]. Patients had to have a red swollen foot with increased local temperature of more than $2^{\circ} \mathrm{C}$ compared with the contralateral foot with x-ray evidence of acute CF. Foot temperature was measured using FLUKE Ti32 thermal imager (Fluke Corporation, WA, USA). All patients with acute CF had neuropathy, diagnosis of which was based on the vibration perception threshold (Neurothesiometer NU-1, Horwell, Neurothesiometer, London, UK) on the big toe being $>25 \mathrm{~V}$ [9]. Among those, five had dislocations or subluxations, four had fractures, five had periosteal reactions and four had bone destruction upon $\mathrm{x}$-ray.

The study was approved by the institutional review boards of Weill Cornell Medicine (WCM), Qatar and HMC (13-00031 and 14-14054, respectively). All participants provided written informed consent. The study was conducted in accordance with the 1964 Declaration of Helsinki and was registered at clinicaltrials.gov (NCT02316483).

\section{Monocytes isolation \& DNA/RNA extraction}

$10 \mathrm{ml}$ of blood was withdrawn from peripheral venous puncture from each participant. Peripheral blood mononuclear cells (PBMCs) were first isolated from whole blood and stained with the mouse antihuman IgG2b CD14-APC and the mouse antihuman IgG1 CD16-PE (BD Bioscience, CA, USA). Monocytes were then sorted using FACSAria2 flow cytometer (BD Biosciences). Purity of the sorting was controlled after each sorting (Supplementary Figure 1). DNA and RNA from monocytes were extracted (Allprep DNA/RNA mini kit, Qiagen, Hilden, Germany) and stored at $-80^{\circ} \mathrm{C}$, then shipped to the epigenomics core at WCM and the New York Genome Center for sequencing.

\section{Enhanced reduced representation bisulfite sequencing \& data processing}

Enhanced reduced representation bisulfite sequencing (ERRBS) libraries, sequencing, data alignment and methylation calls were generated at the Epigenomics Core at WCM as described in Garrett-Bakelman et al. [10]. The published protocol was modified as follows: samples were size selected on a $2 \%$ agarose cassette using a Pippin HT (Sage Science, MA, USA), and two size fragment lengths of 240-375 bp and 375-550 bp were recovered 


\begin{tabular}{|c|c|c|c|c|c|c|c|}
\hline & \multicolumn{2}{|c|}{$\begin{array}{l}\text { Type } 2 \text { diabetes with Charcot foot (18 } \\
\text { subjects, } 5 \text { females) }\end{array}$} & \multicolumn{2}{|c|}{$\begin{array}{l}\text { Type } 2 \text { diabetes with neuropathy (18 } \\
\text { subjects, } 5 \text { females) }\end{array}$} & \multicolumn{2}{|c|}{$\begin{array}{l}\text { Type } 2 \text { diabetes without neuropathy } \\
\text { (18 subjects, } 5 \text { females) }\end{array}$} & \multirow{2}{*}{$\begin{array}{l}\text { Group comparison } \\
\text { p-value }\end{array}$} \\
\hline & Mean (SD) & Range & Mean (SD) & Range & Mean (SD) & Range & \\
\hline $\begin{array}{l}\text { Number of CpG } \\
\text { sites that map to a } \\
\text { gene }^{\dagger}\end{array}$ & $1,099,669(44,842)$ & $\begin{array}{l}1,000,416- \\
1,168,307\end{array}$ & $1,141,969(56,389)$ & $\begin{array}{l}1,040,115- \\
1,213,237\end{array}$ & $1,085,492(65,237)$ & $886,300-1,193,351$ & $6.5 \times 10^{-3}$ \\
\hline $\begin{array}{l}\text { Number of total } \\
\text { CpG sites with } \\
\text { coverage of at } \\
\text { least } 10\end{array}$ & $\begin{array}{l}2,706,340 \\
(113,051)\end{array}$ & $\begin{array}{l}2,546,276- \\
2,902,798\end{array}$ & $\begin{array}{l}2,778,313 \\
(192,791)\end{array}$ & $\begin{array}{l}2,441,023- \\
3,008,473\end{array}$ & $\begin{array}{l}2,595,268 \\
(180,000)\end{array}$ & $\begin{array}{l}2,036,173- \\
2,843,919\end{array}$ & $1.1 \times 10^{-2}$ \\
\hline $\begin{array}{l}\text { Number of genes } \\
\text { with mapped CpG } \\
\text { sites }\end{array}$ & $28,729(620)$ & $27,967-30,278$ & $28,784(1397)$ & $25,546-31,268$ & $29,812(1111)$ & $28,638-33,160$ & $6.2 \times 10^{-3}$ \\
\hline
\end{tabular}

$\dagger$ Methylation of a gene was calculated as the mean (weighted by coverage) observed CpG sites within $2 \mathrm{~kb}$ upstream and $2 \mathrm{~kb}$ downstream of transcription start site, as determined by Ensembl annotation. The bottom portion of the table provides summary statistics of the number of $\mathrm{CpG}$ sites within the mapping interval of a gene, and the total number of $\mathrm{CpG}$ sites with coverage of at least 10 (sites with coverage less than 10 were not used).

In order to identify methylation differences specific to Charcot foot, circulating monocytes were isolated from blood of patients with diabetes but no neuropathy (D), diabetes with neuropathy (DN) and diabetes with both neuropathy and Charcot foot (DCh). CpG methylation data was produced by ERRBS.

ERRBS: Enhanced reduced representation bisulfite sequencing; SD: Standard deviation.

and further processed. Samples were checked for quality via two methods: the distribution of CpG site coverage (Supplementary Figure 2A); experiments that are suffering from PCR duplication bias will have a secondary peak to the right of the primary peak; and distribution of methylation $\beta$-values (Supplementary Figure 2B); this histogram should have a peak toward zero methylation and a peak towards methylation of one [11].

Two analysis approaches were carried out:

- CpG site analysis: for each of the 3.1 million target $\mathrm{CpG}$ sites sequenced, the coverage depth of bases with phred-scaled quality score above 30 (Q30) was quantified. A total of 1,240,581 CpG sites which had Q30 coverage of at least ten times in all patients were kept for comparative analysis. Site methylation from 0 to 1 at these sites was combined into a single file with a row for each site and a column for each individual. Statistical analysis comparing groups is described below.

- Gene-mapped analysis: for each gene, $\mathrm{CpG}$ sites within an interval $2 \mathrm{~kb}$ upstream and $2 \mathrm{~kb}$ downstream of the transcription start site (taken from ENSEMBL annotation) were used to determine a methylation level for the gene since a majority of $\mathrm{CpG}$ islands are within $2 \mathrm{~kb}$ of transcription start site [12]. The methylation of a gene was calculated as the mean methylation of measured sites within the interval, weighted by the coverage at each site [13]. In total, 21,634 genes had at least one CpG site within the mapping interval for all patients. More information regarding $\mathrm{CpG}$ sites and mapping to genes can be found in Table 2.

DNA from circulating monocytes was sequenced in three distinct batches. In order to determine whether a batch effect should be considered in subsequent analysis, the first two principal components of the gene-mapped CpG data were plotted and colored by batch (Supplementary Figure 2C \& D). It was determined that batch had affected the methylation measurements and should therefore be accounted for in statistical analysis.

\section{Differential methylation}

Patients were placed in one of three groups:

- D: group of patients with diabetes but no neuropathy $(\mathrm{n}=18)$

- DN: group of patients with diabetes and neuropathy but no CF $(\mathrm{n}=18)$

- DCh: group of patients with diabetes and CF ( $\mathrm{n}=18)$

In order to test for methylation differences that were specific to $\mathrm{CF}$, patients were also grouped as either having $\mathrm{CF}$ (DCh) or not having CF (DDN; $\mathrm{n}=36$ ). DDN grouping is of primary interest for two reasons: it will tend to identify genes that are different due to CF (which involves neuropathy) without identifying genes that are different due to purely neuropathic reasons and it will provide more total samples, and therefore greater statistical power, than testing for differences between CF and either one of the non-CF groups. This is true as long as the two subgroups being grouped together are not significantly different from each other. Therefore, in order to statistically 
justify this grouping, differential methylation was also tested for the three possible pairings of the three groups: $\mathrm{D}$ versus DN, D versus DCh and DN versus DCh (Supplementary Figures $3 \& 4$ ). If methylation differences are detected in the $\mathrm{D}$ versus $\mathrm{DCh}$ and $\mathrm{DN}$ versus $\mathrm{DCh}$ comparisons but not in the $\mathrm{D}$ versus $\mathrm{DN}$ comparison, then grouping non-CF patients together for comparison with CF is justified.

Singular value decomposition [14] was performed for both methylation of the 1,220,216 autosomal CpG sites and methylation of the 21,049 autosomal genes. Sex chromosomes were excluded just for this portion of the analysis so that the samples did not stratify based on gender. The left-singular vectors represent independent methylation features that can capture large amounts of variance from the original data. Since the left-singular vectors capture much of the variance from the original data and represent a signal from many genes, they can be considered a good proxy for the whole methylome (WM). In order to test for a difference in group methylomes, the first three left-singular vectors from (Figure 1B \& D) were regressed on covariates (batch) and grouped using multivariate linear regression (this was done separately for the CpG site and gene-mapped approaches), which tests for a multivariate difference between groups. Next, all 1,240,581 CpG sites and 21,634 individual genes were tested for differential methylation by regressing a site/gene on covariates (batch) and group using an analogous univariate linear regression methodology, which tests for a univariate difference between group means. Both WM differential methylation and individual site/gene differential methylation for both CpG site and gene-mapped methylation were tested for four groupings of patients: DDN/DCh, D/DN, D/DCh and DN/DCh. p-values were produced using a likelihood ratio test of the model-containing group versus the model-not-containing group. Linear regression has been shown to possess similar statistical power to Wilcoxon rank sum test, Kolmogorov-Smirnov test, permutation test, empirical Bayes method and bump hunting method in simulated DNA methylation studies with sample sizes greater than $n=12$ in each group [15]. Significance was assessed using a false discovery rate of $\alpha=0.05$ for both Bonferroni and Benjamini-Hochberg (BH) multiple test correction procedures.

In order to compare the differential methylation findings to what would be expected by chance, each differential methylation analysis was carried out an additional ten times on permuted data. A sample's group status is permuted in order to disrupt any association between group and methylation. The association between methylation and batch is maintained since this association is required for appropriate covariate correction. These permutation analyses give a sense empirically of how far our original differential methylation p-values are from what we would observe if there were truly no association between group and methylation [16]. The results of this comparison between original and permuted data are displayed in quantile-quantile (QQ) plots (Supplementary Figure 5).

\section{Gene expression data \\ Data generation \& fltering}

DNA was synthesized from 10 ng of good quality total RNA (RIN >7) using SMART-SEQ v4 Ultra Low Input RNA Kit (ClonTech, CA, USA) at New York Genome Center according to the manufacturer's protocol with eight cycles of amplification. Resulting cDNA was cleaned up with a 1:1 volume ratio of AMPURE XP beads (Beckman Coulter, CA, USA) and evaluated on the Fragment Analyzer using a High Sensitivity DNA Assay (AATI). Full-length cDNA was sheared to an average size of $350 \mathrm{bp}$ fragments using Adaptive Focused Acoustics technology (Covaris, MA, USA, LE220). Illumina-compatible libraries were prepared with KAPA Hyper Prep Kit (Roche, Basel, Switzerland) and Illumina dual indexed adapters according to the manufacturer's specifications with nine cycles of amplification. The libraries were quantified by picogreen assay and NGS assay (Fragment Analyzer, AATI) and sequenced on an Illumina HiSeq2500 sequencer ( $\mathrm{v} 4$ chemistry, v2 chemistry for Rapid Run) using two 50 bp cycles.

\section{RNA-sequencing analysis}

The reads were aligned with STAR (version 2.4.0c), and genes annotated in Gencode v18 were quantified with featureCounts (v1.4.3-p1). All the genes with less than one read across all the samples were not taken into consideration, which resulted with a total set of 16,007 genes for 30 samples. Normalization of expression was performed using the Bioconductor package DESeq2 using the rlogTransformation function [17].

\section{Association between DNA methylation \& gene expression in CF patients}

We tested the association between DNA methylation (independent variable) and $\log _{2}$ transformed gene expression (dependent variable) using a linear model in which diseas statuses and batch effects were used as covariates. We included only $\mathrm{BH}$ significant genes on a methylome-wide level (Table 3; $\mathrm{n}=2488$ genes) against their transcripts 


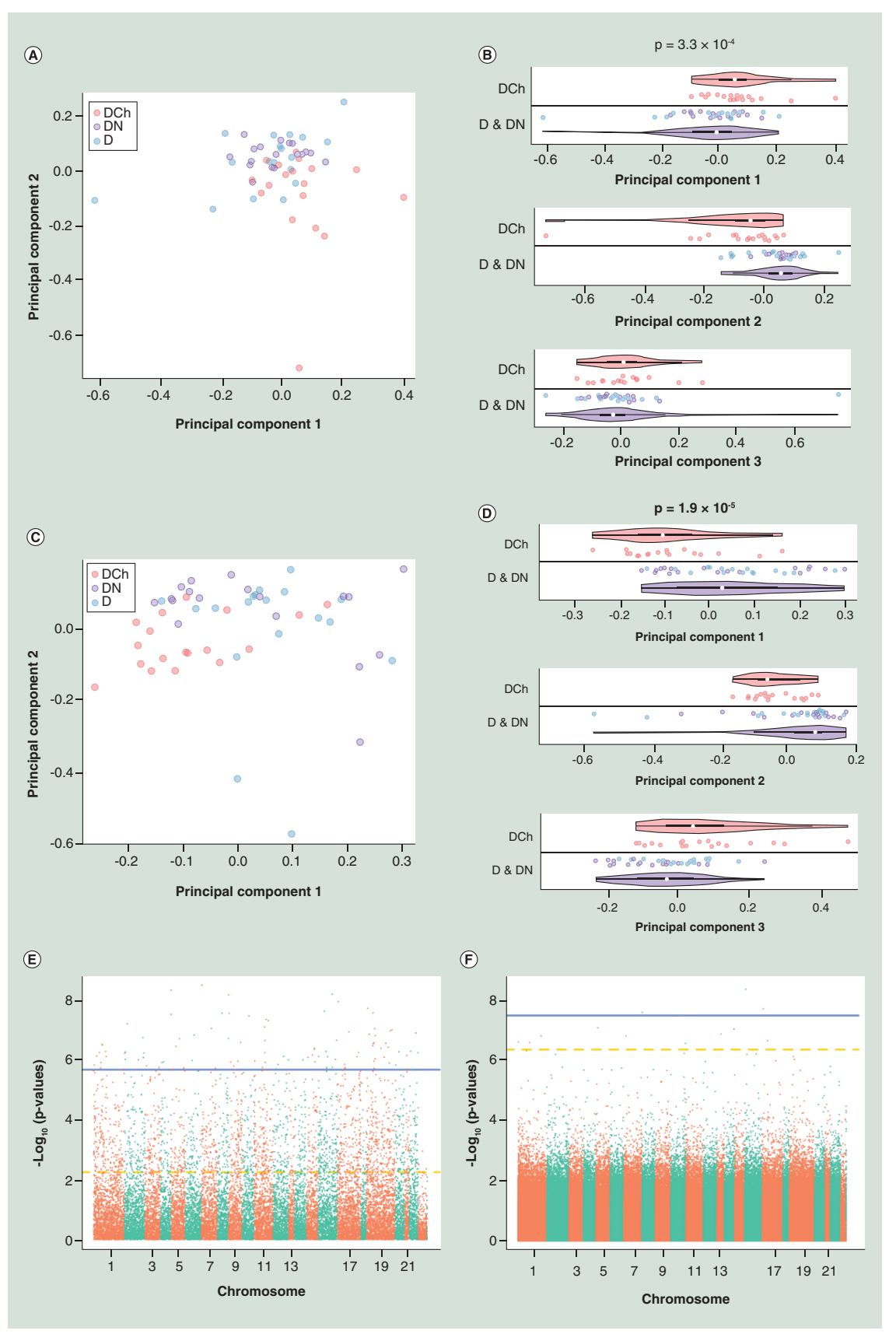

Figure 1. CpG site and gene-mapped differential methylation in patients with diabetes and Charcot foot compared with patients with diabetes but no Charcot foot. (A) The first two principal components of autosomal gene methylation, as calculated by singular value decomposition. Samples are colored by group: diabetes in blue, diabetes with neuropathy in purple and diabetes with CF in red. (B) Whole-methylome signal as captured by the first three principal components (horizontal axes) of the displayed subset of patients. Violin plots representing the distribution of a particular patient group along a principal component are adjacent to their corresponding group. p-value in Figures 1B \& D are assessing the null hypothesis that DCh and ' $D+D N$ ' groups have the same mean methylation in the 3D space, defined by the first three PCA eigenvectors. Given the significant p-values, we are able to reject the null hypothesis that these two groups have the same mean methylation in this reduced dimensional space. (C) The first two principal components of autosomal gene methylation, as calculated by singular value decomposition. Samples are colored by group: diabetes in blue, diabetes with neuropathy in purple and diabetes with CF in red. (D) Whole-methylome signal as captured by the first three principal components (horizontal axes) of the displayed subset of patients. Violin plots representing the distribution of a particular patient group along a principal component are adjacent to their corresponding group. (E) Chromosomal distribution of gene methylation differences. For each gene, the significance is displayed on the $y$-axis as the - $\log _{10}$ of the $\mathrm{p}$-value. The results are ordered along the $\mathrm{x}$-axis by chromosome, with each bar representing a different chromosome. The Bonferroni and Benjamini-Hochberg $p$-value thresholds $(\alpha=0.05)$ were $2.3 \times 10^{-6}$ (yellow line) and $5.8 \times 10^{-3}$ (green dashed line), respectively. (F) Chromosomal distribution of gene methylation differences. For each gene, the significance is displayed on the $y$-axis as the - $\log _{10}$ of the $p$-value. The results are ordered along the $x$-axis by chromosome, with each bar representing a different chromosome. The Bonferroni and Benjamini-Hochberg p-value thresholds $(\alpha=0.05)$ were $4.0 \times 10^{-8}$ (yellow line) and $5.2 \times 10^{-7}$ (green dashed line), respectively.

CF: Charcot foot; D: Group of patients with diabetes but no neuropathy; DCh: Group of patients with diabetes and CF; DN: Group of patients with diabetes and neuropathy but no CF; PCA: Principal component analysis. 
Table 3. Differential methylation results for four groupings of patients.

\begin{tabular}{|llllll|}
\hline Analysis approaches & DDN/DCh & D/DN & D/DCh & DN/DCh & $3.9 \times 10^{-3}$ \\
\hline CpG-site & Methylome-wide p-value & $3.3 \times 10^{-4}$ & 0.92 & $1.0 \times 10^{-2}$ & 0 \\
\hline $\begin{array}{l}\text { Bonferroni-significant } \\
\text { sites }\end{array}$ & 4 & 0 & 0 & 0 \\
\hline Gene-mapped & BH-significant sites & 13 & 0 & 0 & $3.2 \times 10^{-4}$ \\
\hline & Methylome-wide p-value & $1.9 \times 10^{-5}$ & $9.6 \times 10^{-2}$ & 19 & 11 \\
\hline $\begin{array}{l}\text { Bonferroni-significant } \\
\text { genes }\end{array}$ & 114 & 1 & 1494 & 1052 \\
\hline
\end{tabular}

Results of four differential methylation approaches: methylome-wide differential methylation ( $p$-value derived from multivariate linear regression fit using the first three principal components as dependent variables as well as batch and group as independent variables) and individual site/gene differential methylation for both CpG site (top 3 rows) and gene-mapped methylation (bottom 3 rows). For each approach four group comparisons were made, represented by the four columns of the table which are labeled based on the two patient groups that were compared.

BH: Benjamini-Hochberg;D: Group of patients with diabetes but no neuropathy; DCh: Group of patients with diabetes and CF; DN: Group of patients with diabetes and neuropathy but no CF.

in our linear regression analysis. Since a total of 1326 genes were common in both datasets, we performed pvalue correction based on $\mathrm{BH}$ criteria for 1326 genes. Subsequently, to identify potential trans effects between methylation and expression in CF, as well as to identify all the other associations relevant to CF condition, we performed multiple test corrections including all methylated and all expressed genes. As we did not identify a large number of significant $\mathrm{CpG}$ sites at methylome-wide level, only methylation-expression association on gene level were considered in these analyses. Gene ontology (GO) enrichment analysis [18] was performed using the STRING database. STRING is a database of known and predicted protein-protein interactions, direct (physical) and indirect (functional) associations [19].

\section{Results}

\section{Combining diabetic patients with \& without neuropathy}

This analysis consisted of four ways of assessing differential methylation, produced by all combinations of two different ways of looking at methylation (individual CpG site methylation and gene-mapped methylation) and two different ways of looking at differences in methylation (WM and individual site/gene). For each way of assessing differential methylation, four comparisons were carried out: DDN/DCh, D/DN, D/DCh and DN/DCh. The first grouping provides the primary result of interest, since it will identify differential methylation that is specific to $\mathrm{CF}$ and not due to either diabetes or the neuropathy that can afflict acute CF disease. However, in order to justify this grouping, it needs to be shown that diabetic patients with and without neuropathy can be considered similar enough to group together. The last three comparisons are therefore used to establish the similarity of the D and $\mathrm{DN}$ groups relative to the differences evident in both the $\mathrm{D} / \mathrm{DCh}$ and $\mathrm{DN} / \mathrm{Dch}$ comparisons.

Table 3 shows that the D/DN comparison identified one differentially methylated gene, zero differentially methylated CpG sites and was not able to stratify the top three principal components in either the CpG site $(\mathrm{p}=0.92)$ or gene-mapped $\left(\mathrm{p}=9.6 \times 10^{-2}\right.$; insignificant after multiple test correction) approaches. On the other hand, the $\mathrm{D} / \mathrm{DCh}$ and $\mathrm{D} / \mathrm{DN}$ comparisons identified several Bonferroni significant and several hundred BH significant differentially methylated genes, and both comparisons were able to stratify the top three principal components in both approaches (Table 3 and Supplementary Figure 6). These results demonstrate that patients with diabetes are not significantly different to patients with diabetes and neuropathy when assessed for differential methylation in circulating monocytes using the discussed methods. Therefore, it is valid to group D and DN together to use as a single group to compare with CF patients using the same methods that were used to establish similarity of the non-CF patients.

\section{Comparing diabetic patients with \& without neuropathy to patients with CF}

WM analysis (which looks at linear combinations of many CpG sites or genes) demonstrates that CF monocytes are differentially methylated when compared with non-CF monocytes (DDN/DCh) in both the $\mathrm{CpG}$ site (Figure $1 \mathrm{~A}$ $\&$ B) and gene-mapped (Figure 1C \& D) approaches. p-values presented on Figure 1B \& D are assessing the null hypothesis that $\mathrm{DCh}$ and $\mathrm{D}+\mathrm{DN}$ have the same mean methylation in the $3 \mathrm{D}$ space defined by the first three principal component analysis (PCA) eigenvectors. Given the significant p-values, we can reject the null 
hypothesis that these two groups have the same mean methylation in this reduced dimensional space. Individual genes reinforce this result with 114 out of 21,634 genes having p-values lower than the Bonferroni-significant cutoff of $\mathrm{p}=2.3 \times 10^{-6}$ for DDN/DCh (Figure 1E and Supplementary Figure 5E). In total, 13 out of 1,240,581 individual CpG sites had $\mathrm{p}$-values lower than the BH cutoff of $\mathrm{p}=5.2 \times 10^{-7}$ for DDN/DCh (Figure $1 \mathrm{~F}$ and Supplementary Figure 5A). This particular way of assessing differential methylation suffered from a very stringent multiple test correction since over a million CpG sites were tested individually. From a theoretical standpoint, power to detect a given effect size decreases as a researcher performs more tests to identify the effect. Therefore, it makes sense that fewer individual CpG sites than genes were identified as significantly differentially methylated.

Permutation analysis demonstrates that the effect of group on methylation vanishes when group is permuted. This analysis allows the structure of covariates with respect to methylation to remain intact while disrupting any association between group and methylation. These permutation analyses can be thought of as a way of accessing the empirical null distribution, which is not always guaranteed to match the theoretical null distribution [16]. When the empirical null p-value distribution matches the theoretical null p-value distribution (uniform) or is skewed towards 1, the true empirical p-values are expected to be at least as low as the p-values that were calculated. All eight QQ plots (Supplementary Figure 5) show that permutation analyses return p-values either matching the uniform distribution or skewed towards 1 (this is represented in the plots as the cyan line being below the dashed gray line). This result serves to justify the statistical methodology that was used. The QQ plots (Supplementary Figure 5B $\& \mathrm{~F}$ ) also serve as a way of further confirming that $\mathrm{D}$ and $\mathrm{DN}$ do not have different methylation patterns since inspection reveals that the original p-values overlap with the permutation p-values.

The differential methylation of CF compared with non-CF foot using both CpG site and gene-mapped approaches also demonstrate that the way methylation was mapped to genes is at least as effective at capturing discriminatory information as looking at individual $\mathrm{CpG}$ sites. This is not a reference to the number of significant sites or genes identified, instead it has to do with the actual p-values. Comparing Figure $1 \mathrm{E} \& \mathrm{~F}$, it is evident that the mapped approach is producing p-values at least as low as those produced from the $\mathrm{CpG}$ site approach. This finding matters because it means that this approach has largely been able to avoid the pitfall of combining discordant signals from nearby CpG sites, which would be detrimental to the discrimination task. Therefore, we focused the rest of the analysis on the gene-mapped differential methylation.

\section{Gene mapped differential methylation in patients with diabetes \& CF compared with patients with diabetes but no CF}

In total, 114 out of 21,634 genes were differentially methylated in CF patients compared with non-CF monocytes when looking at the gene-mapped methylation (Supplementary Table 1). When we take a closer look to the 114 genes one by one, we notice that 37 genes out of the 114 Bonferroni-significant genes differently methylated for $\mathrm{CF}$ versus non-CF have an unknown function. Most of the 114 genes presented a hypermethylation (86\%) in CF patients (Figure 2A). Figure 2B represents the ten top hypermethylated (top part, red) and hypomethylated (bottom part, green) genes in CF patients.

IPA global analysis of the 114 Bonferroni-significant genes differently methylated for CF versus non-CF revealed significant enrichment of the category presented in the supplementary Figure 7. We showed a more detailed network of two subsets of them: the 'amino acid metabolism, molecular transport, small molecule biochemistry' category (Figure 2C), as well as the category involving 'embryonic development, organismal development, tissue morphology' (Figure 2D). This observation indicates that circulating monocytes in CF patients seem to be 'ready' for differentiation. Both enriched classes are coherent with the experimental design.

The genes differently methylated for CF versus non-CF that could affect this pathway, and therefore be indirectly involved in monocyte differentiation into osteoclasts, are presented in the Supplementary Table 2. A total of 23 genes could be involved directly or indirectly in monocyte differentiation into osteoclasts, which represents around $15 \%$ of the total genes differentially methylated in CF patients (and around 30\% of the genes with known function). One of the top hits in this list is MAPK11 that is known to enhance osteoclastogenesis and bone resorption in breast cancer [20].

\section{Association between DNA methylation \& gene expression in CF patients}

Abnormal DNA methylation can result in aberrant gene expression [21]. Therefore, we investigated gene expression in our samples and try to associate it with the DNA methylation. First, we identified 2488 significant genes with $\mathrm{BH}$ correction $(\mathrm{BH}$ cutoff $=0.0057)$. Of these 2488,818 genes were downregulated and 1670 upregulated 
(A)

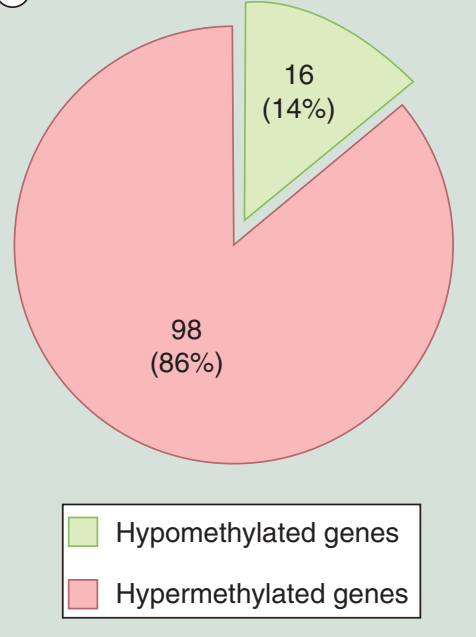

(B)

\begin{tabular}{llr|lll}
\hline \multicolumn{1}{|c}{ Gene name } & p-value & $\begin{array}{c}\text { Log2 fold } \\
\text { change }\end{array}$ & Gene name & p-value & $\begin{array}{c}\text { Log2 fold } \\
\text { change }\end{array}$ \\
\hline WDR25 & $1.08 \mathrm{E}-06$ & 3.211 & RP11-394l13.1 & $1.08 \mathrm{E}-06$ & -0.086 \\
AARS2 & $1.62 \mathrm{E}-07$ & 3.155 & P2RY11 & $3.68 \mathrm{E}-07$ & -0.094 \\
ALDOC & $1.96 \mathrm{E}-06$ & 2.201 & GYS1 & $4.11 \mathrm{E}-07$ & -0.154 \\
RP11-192H23.5 & $1.96 \mathrm{E}-06$ & 2.201 & CACTIN & $1.50 \mathrm{E}-07$ & -0.188 \\
RP11-977B10.2 & $1.54 \mathrm{E}-06$ & 1.963 & NKG7 & $1.17 \mathrm{E}-07$ & -0.223 \\
MX1 & $7.69 \mathrm{E}-07$ & 1.865 & RP11-388M20.2 & $8.58 \mathrm{E}-07$ & -0.331 \\
RP11-867G23.4 & $6.23 \mathrm{E}-07$ & 1.826 & PRSS8 & $8.58 \mathrm{E}-07$ & -0.331 \\
RN7SL758P & $8.83 \mathrm{E}-07$ & 1.802 & RP11-1035H13.2 & $2.60 \mathrm{E}-08$ & -0.544 \\
HMGA1 & $1.13 \mathrm{E}-06$ & 1.759 & RP11-307L3.4 & $3.08 \mathrm{E}-08$ & -0.667 \\
ZBED3-AS1 & $3.85 \mathrm{E}-07$ & 1.734 & FAM120A & $1.76 \mathrm{E}-06$ & -0.966 \\
\hline
\end{tabular}

Figure 2. Gene analysis for gene-mapped differential methylation in patients with diabetes and Charcot foot compared with patients with diabetes but no Charcot foot. (A) Graphic representation of the number of hypo- and hypermethylated genes. (B) Representation of the ten top hypermethylated (top part, red) and hypomethylated (bottom part, green) genes in Charcot foot patients compared with patients with diabetes but no Charcot foot. (C \& D) Networks of altered genes mapped differential methylation created by IPA. The hypothetical networks generated by IPA based on the molecular relationships, interactions and pathway associations between the methylated candidate genes are shown in a graphical representation.

IPA: Ingenuity pathway analysis.

(Supplementary Table 3). Gene methylation can have two different types of associations with gene expression: local association (cis) where one differentially methylated gene (A) will have an effect on the exact same gene (A) on expression level or distal association (trans) where one differentially methylated gene (A) will have an effect on a different gene (B) on expression level (Figure 3A). In order to test for cis- and trans-effect of the methylation on gene expression, we performed linear regression for 2488 genes, treating gene expression as dependent variable and methylation as independent variable, using disease status and batch effect as covariates. PPP2R5D was the only gene detected with a cis association with expression. The correlation coefficient beta is 4.4, so expression and methylation of this gene are positively correlated. Both methylation and expression are increasing in CF patients. We further looked at the location of CpGs relative to PPP2R5D gene. We identified 29 CpGs present in this gene, all within its body and none in the promoter region. Six of those sites were highly methylated while the 23 other CpG sites did not differ between the study group and controls as their methylation level was very low ( 0 or close to 0$)$. All six sites were present within introns, including intron 2-3 (CpG position: chr6.42996110, chr6.4300548, chr6.43005487), intron 9-10 (chr6.43008690) and intron 10-11 (chr6.43008760, chr6.43008765). Furthermore, we tested the relation of individual CpG sites with gene expression; it was not statistically significant (Supplementary Table 4).

For the trans association, we identified 27 genes out of 2488 genes with significant changes in methylation between CF and non-CF that were significantly associated with expression in 24 genes. Four (MTCL1P1, ITGAL, DHX40, GFOD2) of these genes were hypomethylated when comparing CF to non-CF samples, while the others were hypermethylated (Supplementary Table 4). We created a minimum network of protein-protein interactions using the $27 \mathrm{CF}$ differentially methylated genes and their 24 expression-associated genes (total of 51 genes) leading to 32 interactions (Figure $3 \mathrm{~B}$ ). Out of those 32 interactions, seven had a positive correlation coefficient beta and 27 a negative one, suggesting suppression of expression associated with hypermethylation or gene overexpression associated with hypomethylation (Supplementary Table 5).

\section{Discussion}

Monocytes are directly involved in diabetics' CF pathogenesis. They are the progenitors of osteoclasts which are the principal cells responsible for bone resorption. Starting from an early monocytic cell, there is a complete cycle which all cells follow. They have to proliferate, fuse and differentiate under the influence of different precursors before they become adult osteoclasts [22]. 


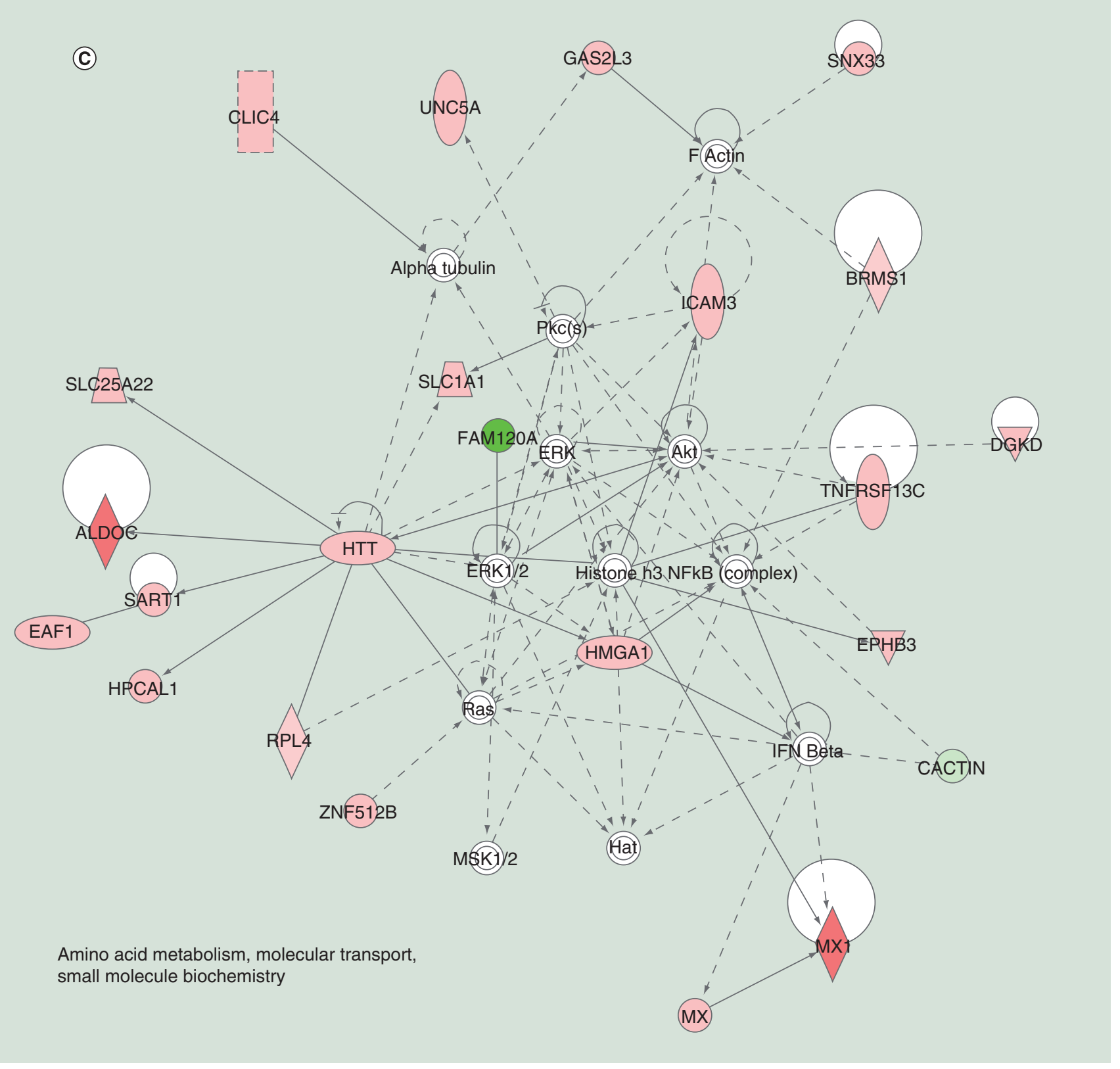

Figure 2. Gene analysis for gene-mapped differential methylation in patients with diabetes and Charcot foot compared with patients with diabetes but no Charcot foot (cont.). (A) Graphic representation of the number of hypo- and hypermethylated genes.

(B) Representation of the ten top hypermethylated (top part, red) and hypomethylated (bottom part, green) genes in Charcot foot patients compared with patients with diabetes but no Charcot foot. (C \& D) Networks of altered genes mapped differential methylation created by IPA. The hypothetical networks generated by IPA based on the molecular relationships, interactions and pathway associations between the methylated candidate genes are shown in a graphical representation.

IPA: Ingenuity pathway analysis.

We have recently demonstrated that five cytokines (G-CSF, GM-CSF, IL-1-ra, IL-2 and IL-16) were significantly increased in circulating microparticles from acute diabetic CF patients, and induced the activation of pathways that are involved in osteoclast formation [4]. Moreover, we revealed the presence of 25 differentially expressed circulating miRNAs in acute diabetic CF that could be involved in monocytes differentiation into osteoclasts [23].

In this study, we showed that methylation of circulating monocytes is involved in the pathogenesis of acute CF. The strength of our study relies on the fact that we used only one cell type from PBMCs. Several reports have already indicated that methylation changes could be cell-specific, and that several variations between cell types exist within 


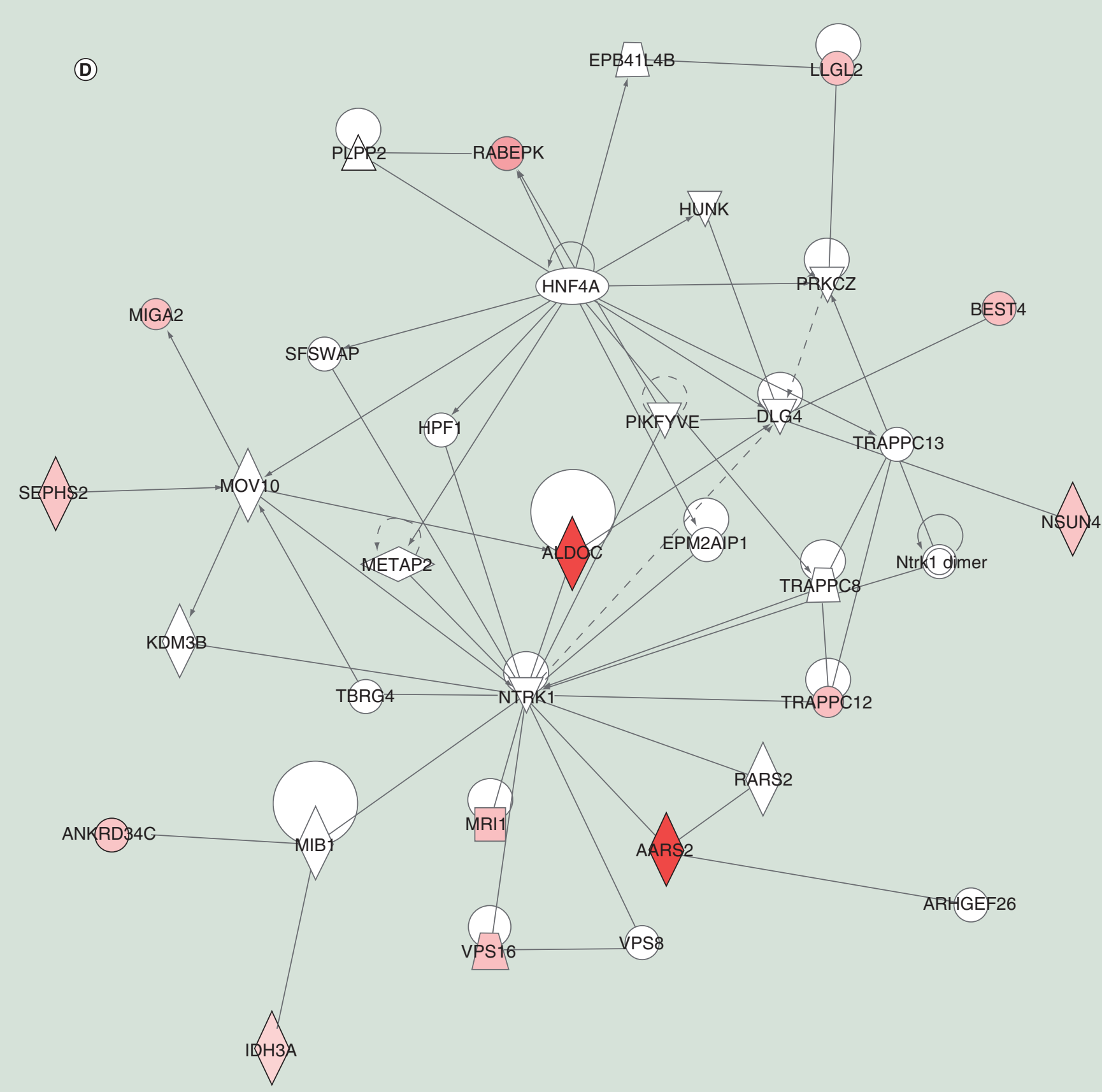

Embryonic development, organismal development, tissue morphology

Figure 2. Gene analysis for gene-mapped differential methylation in patients with diabetes and Charcot foot compared with patients with diabetes but no Charcot foot (cont.). (A) Graphic representation of the number of hypo- and hypermethylated genes.

(B) Representation of the ten top hypermethylated (top part, red) and hypomethylated (bottom part, green) genes in Charcot foot patients compared with patients with diabetes but no Charcot foot. (C \& D) Networks of altered genes mapped differential methylation created by IPA. The hypothetical networks generated by IPA based on the molecular relationships, interactions and pathway associations between the methylated candidate genes are shown in a graphical representation.

IPA: Ingenuity pathway analysis. 
(A)

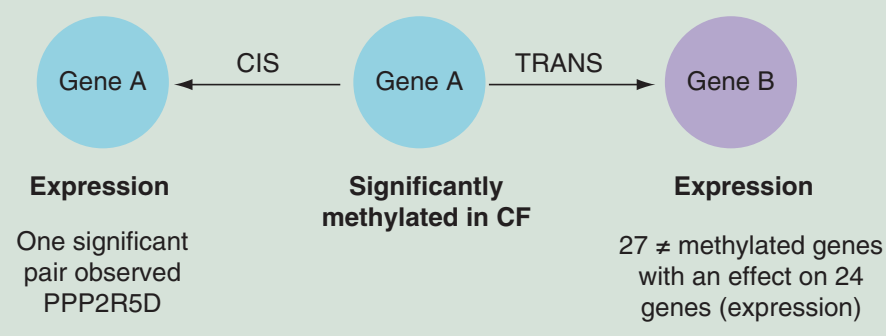

(B)

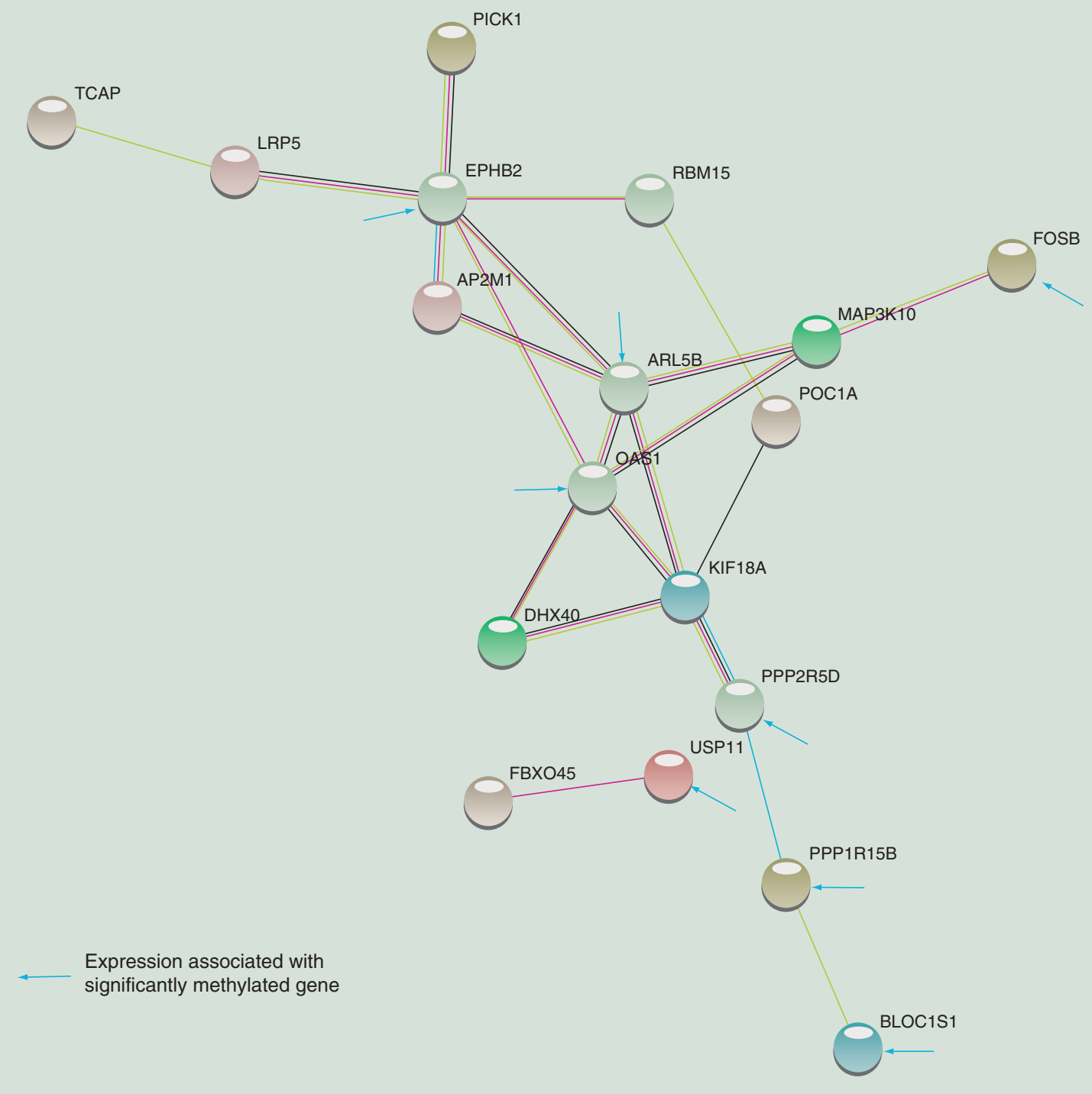

\begin{tabular}{|llll|}
\hline Known interactions & Predicted interactions & Others & \\
$\bigcirc-\bigcirc$ From curated databases & $\bigcirc-\bigcirc$ Gene neighborhood & $\bigcirc$ & $\bigcirc$ Textmining \\
$-\bigcirc$ Experimentally determined & $\bigcirc-\bigcirc$ Gene fusions & $\bigcirc-\bigcirc$ co-expression \\
& $\bigcirc-\bigcirc$ Gene co-occurrence & $\bigcirc \bigcirc$ Protein homology
\end{tabular}

Figure 3. Association between methylation and expression in Charcot foot. (A) Schematic representation of cis- and trans-association between methylated and expressed gene. (B) Minimum network of protein-protein interactions using the 27 CF differentially methylated genes and their 24 expression-associated genes (total of 51 genes), leading to 32 interactions using STRING.

CF: Charcot foot. 
the same individual [24]. Miao et al. [25] demonstrated that monocytes and lymphocytes have distinct epigenomes, whereas patterns within a specific cell type are remarkably similar despite age or gender. It might be therefore inappropriate to assess disease-specific methylation changes using PBMCs as a surrogate end point. Although several reports linked methylation changes to diabetes and its complications, most of them reported differential gene methylation in PBMCs and only a few assessed cell- or tissue-specific methylation changes in diabetes [26]. In pancreatic islets from T2D patients, Volkmar et al. [27] identified 276 CpG loci affiliated to promoters of 254 genes differentially methylated comparing to nondiabetic islets. Nillsson et al. [28] identified over 250 differentially methylated CpG loci in liver tissues of obese T2D patients as compared with nondiabetic individuals. In our study, we showed that the analysis of circulating monocytes WM was not able to differentiate between the groups of D and DN. CF is a neuropathic osteoarthropathy [1], and the fact that monocyte WM could not discriminate D and DN demonstrates that the methylation differences that we uncovered in this study are specific to CF.

We identified several genes that are differentially methylated in circulating monocytes of patients with CF. Furthermore, most of those genes were involved in the migration process of monocytes and their differentiation into osteoclasts. The top hit genes were HMGA1 and MAPK11, both hypermethylated. The important role of P38B (MAPK11) in osteolytic bone destruction has been demonstrated in the context of breast cancer [20]. Upregulation of MCP1 expression by MAPK11 leads to the enhanced osteoclast differentiation and bone resorption. MPAK11 has also been shown to be a regulator of TNF gene expression in mononuclear phagocytes [29]. HMGA1 is a downstream nuclear target of the insulin receptor signaling pathway [30]. HMGA1 is a master regulator of tumor progression by driving inflammatory pathway and cell cycle progression genes during tumorigenesis [31].

To strengthen our methylation data, we performed a transcriptomic study in order to link methylation to gene expression. We demonstrated that only PPP2R5D was significantly methylated and expressed (cis-association) in CF patients. The product of this gene belongs to the phosphatase-2A regulatory subunit B family that is known to be implicated in the negative control of cell growth and division [32]. DNA methylation at promoters is usually associated with decreased gene expression [33], but recent reports suggest that gene body methylation stabilizes transcription, thereby possibly increasing expression [34,35]. In our study, PPP2R5D that had all CpGs present within its body and none in the promoter region was hypermethylated and overexpressed in monocytes of patients with CF, suggesting a decrease of their cell growth. Interestingly, monocytes have to decrease their division in order to differentiate into osteoclasts [36].

The study of trans association revealed $27 \mathrm{CF}$ differentially methylated genes having an effect on 24 expressionassociated genes. Among them, some play an important role in the length of long bones (POC1A) [37] or monocyte trafficking (FOSB) [38]; others are related to glycemic traits in Type 1 diabetes and T2D (OAS1, ARMC7) [39,40], insulin resistance (EPHB2) [41] and Golgi trafficking (ARL5B) [42]. We believe that POC1A and FOSB need to be investigated further in the context of CF disease. In fact, both are involved in bone-related disorders such as short stature, onychodysplasia, bone loss or osteosarcoma, rendering them potential candidate for further functional validation studies in CF disease.

To the best of our knowledge, although recent studies are still using reduced representation bisulfite sequencing (RRBS) [43], we are the first to report differential methylation changes in diabetes and related complications using the ERRBS. RRBS, and related methods such as ERRBS, are sequencing methods that enrich for CpG-rich parts of the genome, which enables sequencing of genomic regions where 5-methylcytosine modifications can alter gene expression $[10,11,44]$. The advantage of this method over whole genome bisulfite sequencing is increased coverage depth and hence higher confidence variant calling for a fixed volume of sequencing reads. With respect to arraybased epigenome genotyping platforms such as the HELP array, ERRBS provides higher sensitivity for rare and population-specific variants $[10,44,45]$. ERRBS has several advantages compared with RRBS; for instance, it increases the biologically pertinent genomic loci covered and has been used to profile cytosine methylation in DNA from human, mouse and other organisms [10]. ERRBS gave a greater number of CpGs represented in the data generated and increased coverage of all genomic regions interrogated.

We acknowledge the presence of few limitations in our study. First, our sample size is relatively small; thus, a higher number of study participants might have enabled us to detect more methylation calls. Additionally, we used circulating monocytes as surrogate markers for CF knowing that the disease is only limited to the foot. Despite the presence of those limitations, we were able to conduct a comprehensive analysis of circulating monocytes methylome in patients with acute diabetic $\mathrm{CF}$ and we demonstrated the presence of differentially methylated genes involved in migration, differentiation and formation of osteoclasts from circulating monocytes. Moreover, we were able to associate the difference in methylation with gene expression. Further studies are needed to determine the 
timing of changes in methylation/expression, whether they precede evidence of acute CF disease or if they could be a downstream effect. Additionally, it would be important to assess whether the differential methylation in the acute stage will be present or not in chronic CF disease, and if those changes are reversible in patients who recover.

\section{Future perspective}

Implications of epigenetics generally, and gene methylation in particular, are being increasingly used in clinical settings. Although drugs that modulate DNA methylation of cancer cells are already used in oncology treatment [46], the pharmaco-epigenetic therapy in T2D and cardiovascular disease is currently limited to experimental studies. For example, inhibition of the methyltransferase SETD7 that is required for DNA methylation in macrophages resulted in a decrease in reactive oxygen species and upregulation of anti-oxidant genes [47]. Similarly, in an experimental model of $\mathrm{db} / \mathrm{db}$ mice with diabetic nephropathy, the angiotensin receptor blocker losartan reverses back the methylation of the histone $\mathrm{H} 3 \mathrm{~K} 9$ that is observed in mesangial cells under hyperglycemia. Our findings could be used to elucidate the cause of CF, with the ultimate goal of finding means to modulate or prevent it. Finally, similar methodology could be used to evaluate the methylome of circulating monocytes in patients with conditions of increased bone resorption.

\section{Summary points}

- Charcot foot disease (CF) is a rare complication of diabetes associated with bone resorption and deformation.

- Osteoclasts are responsible for the bone pathology observed in CF disease, they are derived from monocytes.

- We studied the whole methylome of circulating monocytes using the enhanced reduced representation bisulfite sequencing technique.

- WM analysis demonstrated that CF monocytes are differentially methylated compared with non-CF monocytes, in both CpG-site and gene-mapped analysis approaches.

- Among the methylated genes, several are involved in the migration process during monocytes differentiation into osteoclasts or are indirectly involved through the regulation of inflammatory pathways.

- We also demonstrated an association between methylation and gene expression.

- PPP2R5D was the only gene both differentially methylated and expressed (cis-association) in diabetic CF patients.

- Our findings unveil a possible role of circulating monocytes whole methylome in the pathogenesis of diabetic $\mathrm{CF}_{\text {, }}$ with the ultimate goal being finding means to modulate or prevent it.

\section{Supplementary data}

To view the supplementary data that accompany this paper please visit the journal website at: www.futuremedicine.com/doi/sup $\mathrm{pl} / 10.2217 / \mathrm{epi}-2018-0144$

\section{Author contributions}

Conception and design are made by C Abi Khalil. Acquisition of data is made by J Pasquier, M Spurgeon, M Bradic, B Thomas, A Liberska, J Mezey and J Rodriguez-Flores. The statistical analyses were performed by J Pasquier, M Spurgeon, M Bradic, J RodriguezFlores, J Mezey. O Chidiac, M-J Dib, R Turjoman and C Abi Khalil were involved in the recruiting of the patients. A Robay made sure that this work follows the ethical compliance. Analysis and interpretation of the data were made by J Pasquier, M Spurgeon, M Bradic, M Staudt, KA Fakhro, A Robay, J Rodriguez-Flores, J Mezey, RG Crystal and C Abi Khalil. Figure preparation was done by J Pasquier, M Spurgeon and M Bradic. All authors read and approved the final manuscript. C Abi Khalil is the guarantor.

Financial \& competing interests disclosure

This work was supported by a national research priority program (NPRP) Grant Number 7-701-3-192 from the Qatar National Research Fund (a member of Qatar Foundation). The findings achieved herein are solely the responsibility of the authors. The funder had no role in study design, data collection and analysis, decision to publish, or preparation of the manuscript. The authors have no other relevant affiliations or financial involvement with any organization or entity with a financial interest in or financial conflict with the subject matter or materials discussed in the manuscript apart from those disclosed.

No writing assistance was utilized in the production of this manuscript. 


\section{Acknowledgments}

The authors thank the Flow Cytometry Facility within the Microscopy Core at Weill Cornell Medicine-Qatar for contributing to these studies. The Core is supported by the 'Biomedical Research Program at Weill Cornell Medicine - Qatar,' a program funded by Qatar Foundation.

\section{Ethical disclosure}

All participants provided written informed consent approved by Weill Cornell Medicine-Qatar and Hamad Medical Corporation IRBs (13-00031 and 14-14054, respectively). The study was conducted in accordance with the 1964 Declaration of Helsinki and was registered at clinicaltrials.gov (NCT02316483).

\section{Open access}

This work is licensed under the Attribution-NonCommercial-NoDerivatives 4.0 Unported License. To view a copy of this license, visit http://creativecommons.org/licenses/by-nc-nd/4.0/

\section{References}

Papers of special note have been highlighted as: $\bullet$ of interest; $\bullet \bullet$ of considerable interest

1. Rogers LC, Frykberg RG, Armstrong DG et al. The Charcot foot in diabetes. Diabetes Care 34(9), 2123-2129 (2011).

-• Up-to-date review about Charcot foot disease.

2. Young MJ, Marshall A, Adams JE, Selby PL, Boulton AJ. Osteopenia, neurological dysfunction, and the development of Charcot neuroarthropathy. Diabetes Care 18(1), 34-38 (1995).

3. Mabilleau G, Petrova NL, Edmonds ME, Sabokbar A. Increased osteoclastic activity in acute Charcot's osteoarthropathy: the role of receptor activator of nuclear factor-kappaß ligand. Diabetologia 51(6), 1035-1040 (2008).

4. Pasquier J, Thomas B, Hoarau-Vechot J et al. Circulating microparticles in acute diabetic Charcot foot exhibit a high content of inflammatory cytokines, and support monocyte-to-osteoclast cell induction. Sci. Rep. 7(1), 16450 (2017).

-. Describes the role of microparticles in Charcot foot disease.

5. Udagawa N, Takahashi N, Akatsu T et al. Origin of osteoclasts: mature monocytes and macrophages are capable of differentiating into osteoclasts under a suitable microenvironment prepared by bone marrow-derived stromal cells. Proc. Natl Acad. Sci. USA 87(18), 7260-7264 (1990).

- Describes the differentiation of monocytes into osteoclasts.

6. Bird A. Perceptions of epigenetics. Nature 447(7143), 396-398 (2007).

- Comprehensive review regarding epigenetics.

7. Bird A. DNA methylation patterns and epigenetic memory. Genes Dev. 16(1), 6-21 (2002).

8. Portela A, Esteller M. Epigenetic modifications and human disease. Nat. Biotechnol. 28(10), 1057-1068 (2010).

-. Comprehensive review regarding epigenetics in human disease.

9. Young MJ, Breddy JL, Veves A, Boulton AJ. The prediction of diabetic neuropathic foot ulceration using vibration perception thresholds. A prospective study. Diabetes Care 17(6), 557-560 (1994).

10. Garrett-Bakelman FE, Sheridan CK, Kacmarczyk TJ et al. Enhanced reduced representation bisulfite sequencing for assessment of DNA methylation at base pair resolution. J. Vis. Exp. 96, e52246 (2015).

- Describes enhanced reduced bisulfite sequencing technique.

11. Akalin A, Kormaksson M, Li S et al. methylKit: a comprehensive R package for the analysis of genome-wide DNA methylation profiles. Genome Biol. 13(10), R87 (2012).

12. Zuo T, Liu TM, Lan X et al. Epigenetic silencing mediated through activated PI3K/AKT signaling in breast cancer. Cancer Res. 71(5), 1752-1762 (2011).

13. Akalin A, Garrett-Bakelman FE, Kormaksson M et al. Base-pair resolution DNA methylation sequencing reveals profoundly divergent epigenetic landscapes in acute myeloid leukemia. PLoS Genet. 8(6), e1002781 (2012).

14. Elliott MA, Walter GA, Swift A, Vandenborne K, Schotland JC, Leigh JS. Spectral quantitation by principal component analysis using complex singular value decomposition. Magn. Reson. Med. 41(3), 450-455 (1999).

15. Li D, Xie Z, Pape ML, Dye T. An evaluation of statistical methods for DNA methylation microarray data analysis. BMC Bioinformatics 16, 217 (2015).

16. Efron B. Bayesian inference and the parametric bootstrap. Ann. Appl. Stat. 6(4), 1971-1997 (2012).

17. Love MI, Huber W, Anders S. Moderated estimation of fold change and dispersion for RNA-seq data with DESeq2. Genome Biol. 15(12), 550 (2014). 
18. Zheng Q, Wang XJ. GOEAST: a web-based software toolkit for gene ontology enrichment analysis. Nucleic Acids Res. 36, W358-W363 (2008).

19. Szklarczyk D, Morris JH, Cook H et al. The STRING database in 2017: quality-controlled protein-protein association networks, made broadly accessible. Nucleic Acids Res. 45(D1), D362-D368 (2017).

20. He Z, He J, Liu Z et al. MAPK11 in breast cancer cells enhances osteoclastogenesis and bone resorption. Biochimie 106, 24-32 (2014).

21. Wilson AG. Epigenetic regulation of gene expression in the inflammatory response and relevance to common diseases. J. Periodontol. 79(Suppl. 8), 1514-1519 (2008).

22. Yasuda $\mathrm{H}$, Shima N, Nakagawa $\mathrm{N}$ et al. Osteoclast differentiation factor is a ligand for osteoprotegerin/osteoclastogenesis-inhibitory factor and is identical to TRANCE/RANKL. Proc. Natl Acad. Sci. USA 95(7), 3597-3602 (1998).

23. Pasquier J, Ramachandran V, Abu-Qaoud MR et al. Differentially expressed circulating microRNAs in the development of acute diabetic Charcot foot. Epigenomics doi:10.2217/epi-2018-0052 (2018) (Epub ahead of print).

\section{-. Describes the role of miRNAs in Charcot foot disease.}

24. Zhang B, Zhou Y, Lin N et al. Functional DNA methylation differences between tissues, cell types, and across individuals discovered using the M\&M algorithm. Genome Res. 23(9), 1522-1540 (2013).

25. Miao F, Wu X, Zhang L, Riggs AD, Natarajan R. Histone methylation patterns are cell-type specific in human monocytes and lymphocytes and well maintained at core genes. J. Immunol. 180(4), 2264-2269 (2008).

26. Pasquier J, Hoarau-Vechot J, Fakhro K, Rafii A, Abi Khalil C. Epigenetics and cardiovascular disease in diabetes. Curr. Diab. Rep. 15(12), 108 (2015).

- Comprehensive review regarding epigenetics of diabetes complications.

27. Volkmar M, Dedeurwaerder S, Cunha DA et al. DNA methylation profiling identifies epigenetic dysregulation in pancreatic islets from Type 2 diabetic patients. EMBO J. 31(6), 1405-1426 (2012).

28. Nilsson E, Matte A, Perfilyev A et al. Epigenetic alterations in human liver from subjects with Type 2 diabetes in parallel with reduced folate levels. J. Clin. Endocrinol. Metab. 100(11), E1491-E1501 (2015).

29. Mahlknecht U, Will J, Varin A, Hoelzer D, Herbein G. Histone deacetylase 3, a class I histone deacetylase, suppresses MAPK11-mediated activating transcription factor-2 activation and represses TNF gene expression. J. Immunol. 173(6), 3979-3990 (2004).

30. Chiefari E, Nevolo MT, Arcidiacono B et al. HMGA1 is a novel downstream nuclear target of the insulin receptor signaling pathway. Sci. Rep. 2, 251 (2012).

31. Schuldenfrei A, Belton A, Kowalski J et al. HMGA1 drives stem cell, inflammatory pathway, and cell cycle progression genes during lymphoid tumorigenesis. BMC Genomics 12, 549 (2011).

32. Freeman AK, Dapic V, Monteiro AN. Negative regulation of CHK2 activity by protein phosphatase $2 \mathrm{~A}$ is modulated by DNA damage. Cell Cycle 9(4), 736-747 (2010).

33. Razin A, Cedar H. DNA methylation and gene expression. Microbiol. Rev. 55(3), 451-458 (1991).

34. Yang X, Han H, De Carvalho DD, Lay FD, Jones PA, Liang G. Gene body methylation can alter gene expression and is a therapeutic target in cancer. Cancer Cell 26(4), 577-590 (2014).

35. Singer M, Kosti I, Pachter L, Mandel-Gutfreund Y. A diverse epigenetic landscape at human exons with implication for expression. Nucleic Acids Res. 43(7), 3498-3508 (2015).

36. Tiedemann K, Le Nihouannen D, Fong JE, Hussein O, Barralet JE, Komarova SV. Regulation of osteoclast growth and fusion by $\mathrm{mTOR} /$ raptor and mTOR/rictor/Akt. Front. Cell Dev. Biol. 5 , 54 (2017).

37. Sarig O, Nahum S, Rapaport D et al. Short stature, onychodysplasia, facial dysmorphism, and hypotrichosis syndrome is caused by a POC1A mutation. Am. J. Hum. Genet. 91(2), 337-342 (2012).

38. Kotla S, Singh NK, Kirchhofer D, Rao GN. Heterodimers of the transcriptional factors NFATc3 and FosB mediate tissue factor expression for 15(S)-hydroxyeicosatetraenoic acid-induced monocyte trafficking. J. Biol. Chem. 292(36), 14885-14901 (2017).

39. Go MJ, Hwang JY, Kim YJ et al. New susceptibility loci in MYL2, C12orf51 and OAS1 associated with 1-h plasma glucose as predisposing risk factors for Type 2 diabetes in the Korean population. J. Hum. Genet. 58(6), 362-365 (2013).

40. Qu HQ, Polychronakos C, Type IDGC. Reassessment of the Type I diabetes association of the OAS1 locus. Genes Immun. 10(Suppl. 1), S69-S73 (2009).

41. Kesavan C, Wergedal JE, Lau KH, Mohan S. Conditional disruption of IGF-I gene in type 1alpha collagen-expressing cells shows an essential role of IGF-I in skeletal anabolic response to loading. Am. J. Physiol. Endocrinol. Metab. 301(6), E1191-E1197 (2011).

42. Houghton FJ, Bellingham SA, Hill AF et al. Arl5b is a Golgi-localised small G protein involved in the regulation of retrograde transport. Exp. Cell Res. 318(5), 464-477 (2012).

43. Hao J, Hua L, Fu X, Zhang X, Zou Q, Li Y. Genome-wide DNA methylation analysis of human peripheral blood reveals susceptibility loci of diabetes-related hearing loss. J. Hum. Genet. 63, 1241-1250 (2018). 
44. Gu H, Smith ZD, Bock C, Boyle P, Gnirke A, Meissner A. Preparation of reduced representation bisulfite sequencing libraries for genome-scale DNA methylation profiling. Nat. Protoc. 6(4), 468-481 (2011).

45. Khulan B, Thompson RF, Ye K et al. Comparative isoschizomer profiling of cytosine methylation: the HELP assay. Genome Res. 16(8), 1046-1055 (2006).

46. Jones PA, Issa JP, Baylin S. Targeting the cancer epigenome for therapy. Nat. Rev. Genet. 17(10), 630-641 (2016).

47. He S, Owen DR, Jelinsky SA, Lin LL. Lysine methyltransferase SETD7 (SET7/9) regulates ROS signaling through mitochondria and NFE2L2/ARE pathway. Sci. Rep. 5, 14368 (2015). 